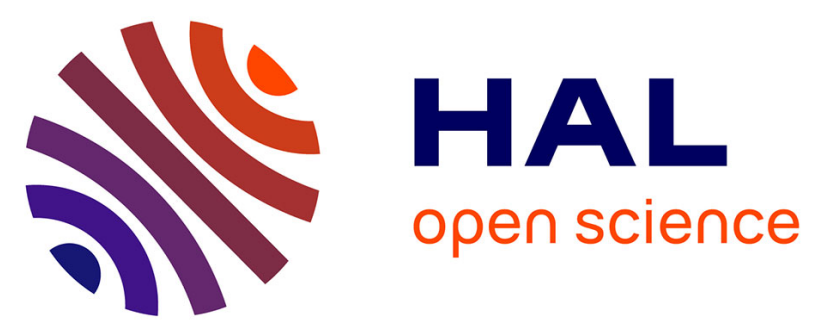

\title{
Evaluation of Intra-Subband Power Allocation for a Downlink Non-Orthogonal Multiple Access (NOMA) System
}

Zeina Eddo, Marie Rita Hojeij, Charbel Abdel Nour, Joumana Farah, Catherine Douillard

\section{To cite this version:}

Zeina Eddo, Marie Rita Hojeij, Charbel Abdel Nour, Joumana Farah, Catherine Douillard. Evaluation of Intra-Subband Power Allocation for a Downlink Non-Orthogonal Multiple Access (NOMA) System. GLOBECOM 2016: IEEE Global Communications Conference: 2nd International Workshop on 5G RAN Design, Dec 2016, Washington, United States. 10.1109/GLOCOMW.2016.7848899 . hal-01439883

\section{HAL Id: hal-01439883 https://hal.science/hal-01439883}

Submitted on 17 Feb 2020

HAL is a multi-disciplinary open access archive for the deposit and dissemination of scientific research documents, whether they are published or not. The documents may come from teaching and research institutions in France or abroad, or from public or private research centers.
L'archive ouverte pluridisciplinaire HAL, est destinée au dépôt et à la diffusion de documents scientifiques de niveau recherche, publiés ou non, émanant des établissements d'enseignement et de recherche français ou étrangers, des laboratoires publics ou privés. 


\title{
Evaluation of Intra-Subband Power Allocation for a Downlink Non-Orthogonal Multiple Access (NOMA) System
}

\author{
Zeina Eddo ${ }^{(1)}$, Marie-Rita Hojeij ${ }^{(1,2)}$, Charbel Abdel Nour ${ }^{(2)}$, Joumana Farah ${ }^{(3)}$, Catherine Douillard ${ }^{(2)}$ \\ ${ }^{(1)}$ Department of Telecommunications, Faculty of Engineering, \\ Holy Spirit University of Kaslik, P.O. Box 446, Jounieh, Lebanon \\ ${ }^{(2)}$ Telecom Bretagne (Institut Mines-Telecom), Department of Electronics, Lab-STICC - UMR CNRS 6285 \\ Technopôle de Brest Iroise, CS 83818 - 29238 BREST Cedex 3, France \\ (3) Department of Electricity and Electronics, Faculty of Engineering, \\ Lebanese University, Roumieh, Lebanon
}

\begin{abstract}
Non-Orthogonal Multiple Access (NOMA) schemes superpose multiple users in the power domain. It is commonly assumed that the achieved throughput in a downlink NOMA system increases with the channel gain difference between paired users. This paper investigates the accuracy of this claim when different intra-subband power allocation techniques are studied. Moreover, the Proportional Fairness (PF) scheduler is used as a resource allocation mechanism to highlight the importance of the choice of user pairing and of the intra-subband power allocation scheme. Results show that the achieved throughput does not always evolve in the same direction as the channel gain difference between paired users, owing to the choice of the intra-subband power allocation technique. In addition, it was found that the PF scheduler is not fully adapted to NOMA.
\end{abstract}

Index Terms - FPA, FTPA, intra-subband power allocation, proportional fairness, non-orthogonal multiple access (NOMA).

\section{INTRODUCTION}

$\mathrm{T}$ WO of the main motivations behind the development of a new generation of mobile networks are the tremendous increase of the wireless data traffic expected to reach 1000 times higher than today's for mobile data volume, and the rise of the number of connected devices that brings out the need to serve almost 300,000 devices per access point [1]. Consequently, one of the challenging $5 \mathrm{G}$ requirements is the increase in the total system capacity that could be met using Multiple Access technology. Non-Orthogonal Multiple Access (NOMA) is a newly emerging access scheme that could serve a new generation of mobile networks by exploiting the power domain for user multiplexing. Thereupon, NOMA takes advantage of the channel gain difference between paired users to allocate the power to every user in an inversely proportional manner to its channel gain [2]. NOMA concept proved that the number of available subbands does not impose a constraint on the number of users that could be served [3].

Part of this work has been performed in the framework of the Horizon 2020 project FANTASTIC-5G (ICT-671660) receiving funds from the European Union. The authors would like to acknowledge the contributions of their colleagues in the project, although the views expressed in this contribution are those of the authors and do not necessarily represent those of the project.
Hence, it can contribute to a $30 \%$ growth of the total system throughput while also improving cell-edge user experience through the attribution of a larger amount of power [2]. By doing so, adopting NOMA implicitly strengthens the fairness between all users located in the same cell, and avoids the underutilization of subbands in the case where a cell-edge user is scheduled alone using Orthogonal Frequency Division Multiple Access (OFDMA) [4]. In addition, this technique brings further advantages such as an improved spectral efficiency without the need to deploy additional transmit antennas, a flexible integration on top of the OFDMA and the Single Carrier-Frequency Division Multiple Access (SCFDMA) for downlink and uplink respectively, and a possible extension to multi-antenna technologies [4].

However, the design of a NOMA system should be carefully addressed to enable a full exploitation of the nonorthogonal multiplexing in the power domain. The total cell throughput, the cell-edge user throughput and the fairness are highly dependent on the way both the power and the bandwidth are being allocated to users, as well as the user pairing for NOMA multiplexing [4]. It is widely agreed that the cell throughput is maximized when the channel gain difference between multiplexed users is high [5], [6], although this statement is questioned in a few number of papers. In [7], the performance of two intra-subband power allocation schemes was evaluated: the first one decides the power levels in a static manner based on the average Signal to Interference and Noise Ratio (SINR) while the second one dynamically attributes the power to users according to the instantaneous SINR. For the fixed scheme, it is found that the gain of downlink NOMA over OFDMA increases when the channel gain difference between multiplexed users increases. As for the dynamic scheme, this difference is limited by some constraints. In [8], the authors underlined that the incorrect choice of the intra-subband power allocation technique or the target data rate leads to a perceived penalty for allocated users in terms of Quality of Service.

Motivated by these contradicting views, we have studied the impact of intra-subband power allocation for NOMA. The 
remainder of this paper is organized as follows: in Section II, the system model is detailed, including the concept of downlink NOMA and an overview of the most used intrasubband power allocation techniques. Then, in Section III, the relationship between the throughput and the channel gain is evaluated. Section IV presents simulation results and Section $\mathrm{V}$ finally concludes the paper.

\section{SYSTEM MODEL}

\section{A. Downlink NOMA Concept}

Consider a single-cell Single-Input Single-Output (SISO) system granted a total bandwidth $B$ for $K$ users to be served, with $B$ being divided into $S$ subbands. From the total set of users $\quad K, \quad$ a subset $U_{s}=\left\{k_{1}, k_{2}, \ldots, k_{n}, \ldots, k_{n(s)}\right\}$, $(1 \leq n \leq n(s))$, is chosen to be multiplexed together on subband $s,(1 \leq s \leq S)$. For each user $k_{n}$ assigned to subband $s$, the Base Station (BS) sends a signal $x_{s, k_{n}}$ with a power $P_{s, k_{n}}$. The multiplexed signal $x_{s}$ transmitted by the BS on subband $s$ is given by

$$
x_{s}=\sum_{n=1}^{n(s)} x_{s, k_{n}}
$$

A maximum transmit power per BS, $P_{\max }$, is imposed and equally divided between subbands such that every subband is granted a power $P_{s}$. Therefore, the power is divided between the multiplexed users such that

$$
P_{\max }=\sum_{s=1}^{S} P_{s}=\sum_{s=1}^{S} \sum_{n=1}^{n(s)} P_{s, k_{n}}
$$

The signal received by user $k_{n}, y_{s, k_{n}}$, can be expressed as

$$
y_{s, k_{n}}=h_{s, k_{n}} x_{s, k_{n}}+w_{s, k_{n}}
$$

where $h_{s, k_{n}}$ is the channel coefficient between user $k_{n}$ scheduled on subband $s$ and its serving BS. $w_{s, k_{n}}$ represents the received Gaussian noise plus inter-cell interference.

At the receiver side, a Successive Interference Cancellation (SIC) process is implemented. It exploits the difference in power introduced by NOMA to help the receiver identify its own signal. The optimal order for user's decoding is in the increasing order of the channel gains observed by users, normalized by the noise, $h_{s, k_{n}}^{2} / N_{s, k_{n}}$, where $h_{s, k_{n}}^{2}$ is the equivalent channel gain and $N_{s, k_{n}}$ the average power of $w_{s, k_{n}}$. Any user $k_{n}$ can remove the inter-user interference from the $j^{\text {th }}$ user, $k_{j}$, whose $h_{s, k_{j}}^{2} / N_{s, k_{j}}$ is lower than $h_{s, k_{n}}^{2} / N_{s, k_{n}}$, and then treats the received signals from other users whose $h_{s, k_{j}}^{2} / N_{s, k_{j}}$ is higher than noise [9]. Consequently, any user can correctly decode the signals of other users that have a normalized channel gain lower than its own.

In [10], the number of non-orthogonally multiplexed users is varied between 2 and 3, and it was shown that the gain brought by the multiplexing of 3 users is largely unjustified compared to the added SIC complexity at the receiver. Hence, in our work, we will limit the number of multiplexed users on the same subband to two, like in [11]. Now, assuming a perfect system where the decoding is successful for all users and no SIC error propagation could occur, the achievable throughput of the user with the highest and the lowest channel gains can be inferred from the channel capacity formula:

$$
R_{s, k_{1}}=B_{s} \log _{2}\left(1+\frac{h_{s, k_{1}}^{2} P_{s, k_{1}}}{N_{s, k_{1}}}\right)
$$

and

$$
R_{s, k_{2}}=B_{s} \log _{2}\left(1+\frac{h_{s, k_{2}}^{2} P_{s, k_{2}}}{h_{s, k_{2}}^{2} P_{s, k_{1}}+N_{s, k_{2}}}\right)
$$

$B_{s}$ is the subband bandwidth, considered equal for all subbands in this work.

As for subband allocation, it will be based on the PF scheduler metric since it is the most used scheduler in NOMA schemes. The PF scheduler was first introduced in [12] for an OMA system, then an adequate metric was developed for NOMA systems in [13]. This scheduler represents a good balance between throughput maximization and user fairness. A set of users $U_{s}$ is chosen among all possible candidate sets $U$ to be scheduled on subband $s$ according to the following metric:

$$
U_{s}=\arg \max _{U} \sum_{k_{n} \in U} \frac{R_{s, k_{n}}(t)}{T_{k_{n}}(t)}
$$

where $R_{s, k_{n}}(t)$ is the feasible throughput for user $k_{n}$ at time slot $t$ and $T_{k_{n}}(t)$ is its historical rate calculated using a moving average window of length $t_{c}$ and updated at every new scheduling slot based on

$$
T_{k_{n}}(t+1)=\left(1-\frac{1}{t_{c}}\right) T_{k_{n}}(t)+\frac{1}{t_{c}} \sum_{s=1}^{S} R_{s, k_{n}}(t)
$$

\section{B. Intra-Subband Power Allocation}

Several power allocation techniques between multiplexed users for NOMA exist in literature [10]. These techniques can be grouped into two main categories: fixed schemes and dynamic schemes. This paper focuses on the Fixed Power Allocation (FPA) and the Fractional Transmit Power Allocation (FTPA).

The Fixed Power Allocation (FPA) technique is the least complex of all methods. The power is divided between the users on a certain subband according to a linearly fixed ratio $\beta$ [10]. In the case of two non-orthogonally multiplexed users on a subband $s$, the total power attributed to this subband, $P_{s}$, is divided according to $\left(\beta P_{s},\left(1-\beta P_{s}\right)\right), \beta(0.5 \leq \beta \leq 1)$ being a constant parameter over all subbands. The user with the highest channel gain is given $\beta P_{s}$ and the paired user is given the rest. FPA allocates the power to users regardless of their 
channel gains. Thus, there is no need to communicate the power attributed to users via signaling since it remains unvaried and it can be easily determined based on the decoding order of users, which in turn reduces the SIC complexity.

The Fractional Transmit Power Allocation (FTPA) is yet another way of dividing the power between multiplexed users on subband $s$, dynamically based on their channel gains.

In the case of two multiplexed users, $P_{s, k_{1}}$ and $P_{s, k_{2}}$ are now attributed as follows [10]:

$$
\begin{aligned}
& P_{s, k_{1}}=\frac{\left(h_{s, k_{1}}^{2} / N_{s, k_{1}}\right)^{-\alpha}}{\left(h_{s, k_{1}}^{2} / N_{s, k_{1}}\right)^{-\alpha}+\left(h_{s, k_{2}}^{2} / N_{s, k_{2}}\right)^{-\alpha}} P_{s} \\
& P_{s, k_{2}}=\frac{\left(h_{s, k_{2}}^{2} / N_{s, k_{2}}\right)^{-\alpha}}{\left(h_{s, k_{1}}^{2} / N_{s, k_{1}}\right)^{-\alpha}+\left(h_{s, k_{2}}^{2} / N_{s, k_{2}}\right)^{-\alpha}} P_{s}
\end{aligned}
$$

$\alpha$ is a factor ranging between 0 and 1 that controls the amount of power attributed to every user; its value is kept constant throughout the power allocation process. FTPA requires the signaling of the power levels to users, increasing its complexity/drawbacks when compared to FPA.

Another dynamic scheme is the Full Search Power Allocation (FSPA). It involves searching among all possible power repartitions and chooses the repartition that achieves the highest throughput [10]. The complexity of this technique is therefore significantly higher compared to FPA and FTPA. Even though FSPA imposes a high penalty in terms of complexity, it was found in [10] that its resulting throughput is only slightly higher than the one achieved by FPA and FTPA. Therefore, targeting an implementable scenario with a good throughput/complexity tradeoff, we do not consider this technique in the rest of this study.

\section{Throughput Evaluation}

\section{A. Channel Gain Difference Dependency}

In this section, we analyze the impact of the channel gain difference between multiplexed users on the achieved throughput for both the FPA and the FTPA techniques. In order to carry out this analysis, we consider the case where the channel gain difference between multiplexed users, $d=h_{s, k_{1}}^{2}-h_{s, k_{2}}^{2}$, is increasing. We assume that, since none of the multiplexed users has been previously chosen, the increase in $d$ results both from an increase in $h_{s, k_{1}}^{2}$ and a decrease in $h_{s, k_{2}}^{2}$.

When FPA is used, $P_{s, k_{1}}$ and $P_{s, k_{2}}$ are constant regardless of the choice of users. In (4), an increase in $h_{s, k_{1}}^{2}$ results in an increase in $R_{s, k_{1}}$ as well. In (5), the decrease of $h_{s, k_{2}}^{2}$ leads to the decrease of both the numerator and the denominator inside the logarithm. However, since $P_{s, k_{2}}>P_{s, k_{1}}$, the whole term inside the log function decreases, leading to the decrease of
$R_{s, k_{2}}$. Nevertheless, since $R_{s, k_{1}}$ is higher than $R_{s, k_{2}}$, the increase in $R_{s, k_{1}}$ is higher than the decrease in $R_{s, k_{2}}$, leading to an increase in the overall achieved rate on every subband $s, R_{s}$. To sum up, when FPA is deployed, $R_{s}$ is an increasing function of $d$.

When FTPA is used, if $h_{s, k_{1}}^{2}$ increases and $h_{s, k_{2}}^{2}$ decreases, $P_{s, k_{1}}$ decreases according to (8) since $h_{s, k_{1}}^{2} \geq h_{s, k_{2}}^{2}$. Thus, the monotony of the product $h_{s, k_{1}}^{2} P_{s, k_{1}}$ in (4) cannot be predicted: it depends on the value of $\alpha$. Therefore, the monotony of $R_{s, k_{1}}$ with $d$ cannot be predicted either. The same applies for $R_{s, k_{2}}$ given by (5). In short, in the case of FTPA, the relationship between the total achieved throughput on every subband $S$ and the channel gain difference between paired users is unpredictable and could evolve either in the same direction or in different directions.

\section{B. Channel Gain Dependency}

Since the behavior of FTPA is not predictable when varying the channel gain values, further analysis is of interest.

We now study the impact of varying the channel gain of $k_{2}$ assuming the user with the highest channel gain, $k_{1}$ is chosen $\left(h_{s, k_{1}}^{2}>h_{s, k_{2}}^{2}\right.$ ). Based on (8) and (4), decreasing $h_{s, k_{2}}^{2}$ makes $P_{s, k_{1}}$ and $R_{s, k_{1}}$ decrease, too. Unfortunately, applying a similar reasoning for $R_{s, k_{2}}$ does not work, since neither the monotony of the numerator in (5) nor the variation of $R_{s}$ with $h_{s, k_{2}}^{2}$ can be directly inferred using such a simple reasoning.

Referring to all the above statements in both sections, we can now conclude that $R_{s, k_{2}}$ has one or more critical points on its curve. These points can be found by calculating the derivative of $R_{s, k_{2}}$ with respect to the channel gain difference $d$, expressed as a function of $P_{s}$ as shown in the following expression:

$$
R_{s, k_{2}}=B_{s} \log _{2}\left(1+\frac{P_{s} h_{s, k_{2}}^{2-2 \alpha}}{P_{s} h_{s, k_{1}}^{-2 \alpha} h_{s, k_{2}}^{2}+N_{s, k_{2}} h_{s, k_{1}}^{-2 \alpha}+N_{s, k_{2}} h_{s, k_{2}}^{-2 \alpha}}\right)
$$

The derivative can be written as:

$$
\frac{d R_{s, k_{2}}}{d\left(h_{s, k_{1}}^{2}-h_{s, k_{2}}^{2}\right)}=\frac{1}{2 h_{s, k_{1}}} \frac{d R_{s, k_{2}}}{d h_{s, k_{1}}}-\frac{1}{2 h_{s, k_{2}}} \frac{d R_{s, k_{2}}}{d h_{s, k_{2}}}
$$

By setting (11) to 0 , one can show that the critical points of (10) verify:

$$
\begin{aligned}
& \alpha P_{s} h_{s, k_{2}}^{2}\left(h_{s, k_{2}} / h_{s, k_{1}}\right)^{2}+\alpha N_{s, k_{2}}\left(h_{s, k_{2}} / h_{s, k_{1}}\right)^{2}+\alpha P_{s} h_{s, k_{2}}^{2} \\
& +(2 \alpha-2) N_{s, k_{2}}=N_{s, k_{2}}\left(h_{s, k_{2}} / h_{s, k_{1}}\right)^{-2 \alpha}
\end{aligned}
$$

The detailed derivation of the proof is given in the appendix at the end of the paper.

Equation (12) shows that the critical points are dependent on $h_{s, k_{1}}^{2}$ and $h_{s, k_{2}}^{2}$. This means that the particular values of the 
channel gains of users play an important role in the throughput variation, and not only their difference.

\section{Simulation Results}

\section{A. Simulation Assumptions}

This section details the system level simulation parameters used in this study, based on existing LTE/LTE-Advanced specifications [14]. A single-cell omnidirectional SISO system is deployed. The cell radius is $500 \mathrm{~m}$ and users are randomly located in every cell with a uniform distribution. Their number varies between 5 and 20. The minimum distance between the user and the base station is set to $35 \mathrm{~m}$. The transmit power per base station is limited to $46 \mathrm{dBm}$. The wireless radio channel is simulated using the Extended Typical Urban (ETU) model. The available bandwidth $B$, divided over 8 subbands, is equal to $10 \mathrm{MHz}$. The developed model accounts for the distance-dependent pathloss and for the frequency-selective Rayleigh fading. The speed of users is taken equal to $50 \mathrm{~km} / \mathrm{h}$ at the carrier frequency of $2 \mathrm{GHz}$. The delay spread is taken equal to $500 \mathrm{~ns}$, and the pathloss is modeled as $P L=128.1+37.6 \log _{10}(r)$ where $r$ is expressed in $\mathrm{km}$ and $P L$

in $\mathrm{dB}$. The noise power spectral density level is assumed to be constant over all subbands, and equal to $4 \times 10^{-18} \mathrm{~mW} / \mathrm{Hz}$. We also assume that the base station perfectly knows the channel gains of its users.

\section{B. Simulation Results}

First, the relationship between the achieved data rate and the channel gain difference between multiplexed users is investigated for each of the two intra-subband power allocation techniques, considering the same assumptions related to the channel gains as in section III.A.

Fig. 1, Fig. 2 and Fig. 3 respectively represent the behavior of $R_{s, k_{1}}, R_{s, k_{2}}$ and $R_{s}$ with respect to the variation of $d$ for the two types of intra-subband allocations.

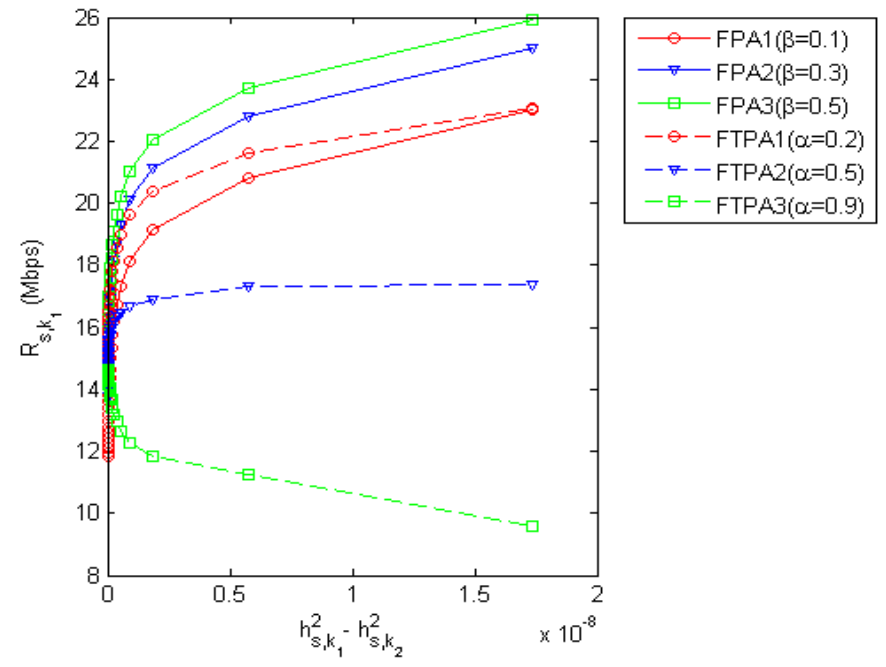

Fig. 1. $R_{s, k_{1}}$ achieved for different FPA and FTPA patterns, as a function of the channel gain difference $d$.
Fig. 1 confirms that $R_{s, k_{1}}$ always increases with $d$ when FPA is used, regardless of the choice of $\beta$. Quite the contrary, when FTPA is used, the choice of $\alpha$ is critical for $R_{s, k_{1}}$ since the same direction of variation is shown for $\alpha=0.2$, and opposite directions for the rest of $\alpha$ values used in the simulations. Moreover, we can see that in the cases where $R_{s, k_{1}}$ is increasing with FTPA, the slope is less steep than with FPA. Thus, FTPA performs better in terms of $R_{s, k_{1}}$ only for very low channel gain differences $\left(d<0.05 \times 10^{-8}\right)$ for any chosen $\alpha$.

The behavior of $R_{s, k_{2}}$ shown in Fig. 2 complies with the analysis of section III.A. When FPA is used, $R_{s, k_{2}}$ is a decreasing function of $d$. However, when FTPA is deployed, the monotony of $R_{s, k_{2}}$ depends on the value of $d$ : a fast increase in $R_{s, k_{2}}$ is obtained at low channel gain differences, but for higher values of $d$, the increase in $R_{s, k_{2}}$ slows down and a decrease is even observed for high values of $\alpha$.

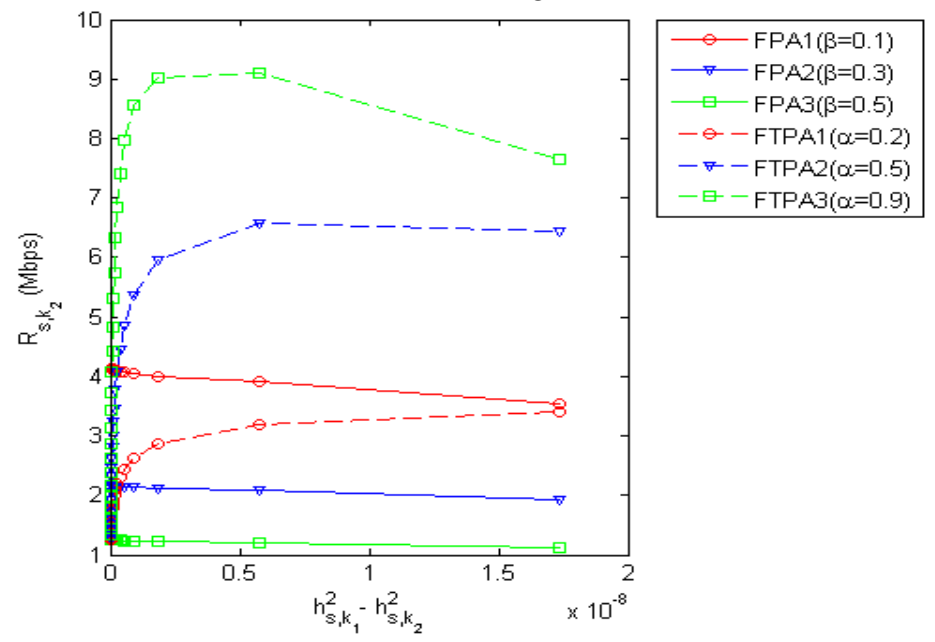

Fig. 2. $R_{s, k_{2}}$ achieved for different FPA and FTPA patterns, as a function of the channel gain difference $d$.
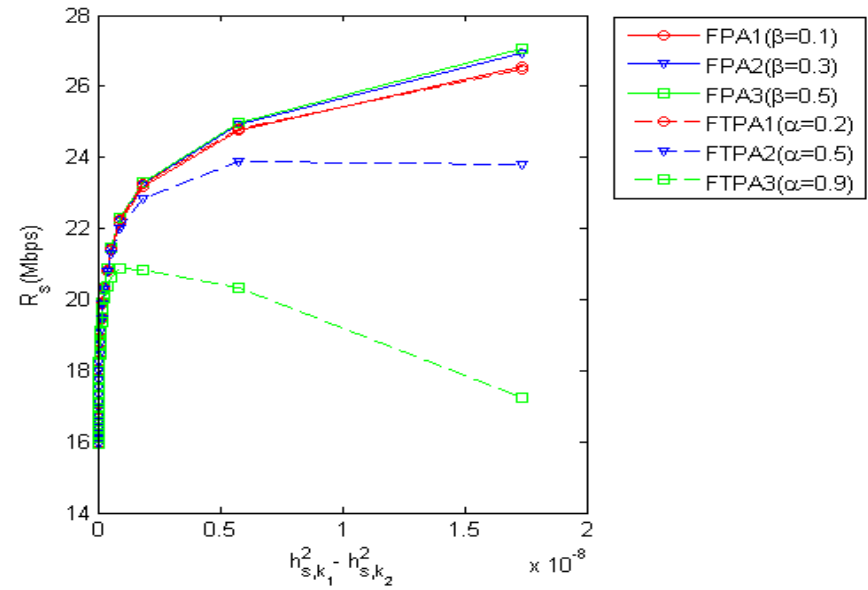

Fig. 3. Overall rate $R_{s}$ achieved for different FPA and FTPA patterns, as a function of the channel gain difference $d$. 
Fig. 3 shows the variations of the overall rate $R_{s}$. We can observe that FPA can achieve a higher overall throughput than FTPA and that, when FTPA is used, the increase in the channel gain difference between scheduled users is not always in favor of NOMA's achieved throughput, contrary to what is commonly assumed.

In order to bring out the impact of the channel gain values on $R_{s, k_{2}}$, Fig. 4 shows a set of curves representing $R_{s, k_{2}}$ as a function of $d$, each curve corresponding to a fixed user $k_{1}$ (and therefore to a fixed value of $h_{s, k_{1}}^{2}$ ) with all the possibilities of pairing with any other user $k_{2}$. Only pattern FTPA2 is considered, but the same behavior was observed for the other FTPA patterns. As already previously observed, for every specific user $k_{1}, R_{s, k_{2}}$ increases with $d$ until reaching a maximum value and then falls. This was already observed in the previous results. However, Fig.4 shows that the critical point from which $R_{s, k_{2}}$ starts to decrease does not depend only on the channel gain difference but also on the choice of user $k_{1}$. The set of critical points, represented by the dashed curve, verify (12) and were proven to be local maxima using the Hessian matrix.

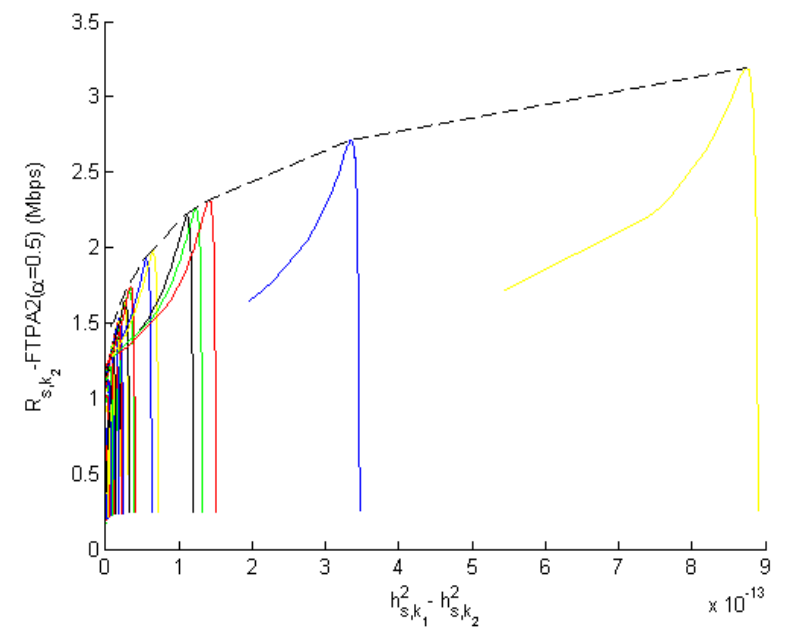

Fig. 4. $R_{s, k_{2}}$ achieved when $h_{s, k_{1}}^{2}$ is fixed and $h_{s, k_{2}}^{2}$ is varied, tested using the FTPA2 pattern.

The last part of our analysis deals with the performance of the PF scheduler when FPA and FTPA are used. Fig. 5 shows the total achieved throughput as a function of the number of users per cell. In contrast with the previous analysis, we can observe that higher overall throughputs can be obtained with FTPA than when applying FPA. This observation remains valid for a high number of users, although an increase in the number of users should naturally lead to the increase in the possible values for $d$. Therefore, additional testing was done in order to investigate how users are being paired together. To this end, statistics were conducted and the results showed that the very large majority of paired users have been chosen with very small channel gain difference values (between 0 and $\left.0.01 \times 10^{-11}\right)$, for both intra-subband allocation techniques.
The result observed in Fig. 5 is thus justified since, for very low channel gain distance values, FTPA can provide higher throughput than FPA (see Fig. 3).

Theoretically, the obtained results can be justified. When FPA is implemented, the data rate varies monotonically with the channel gain difference between users.

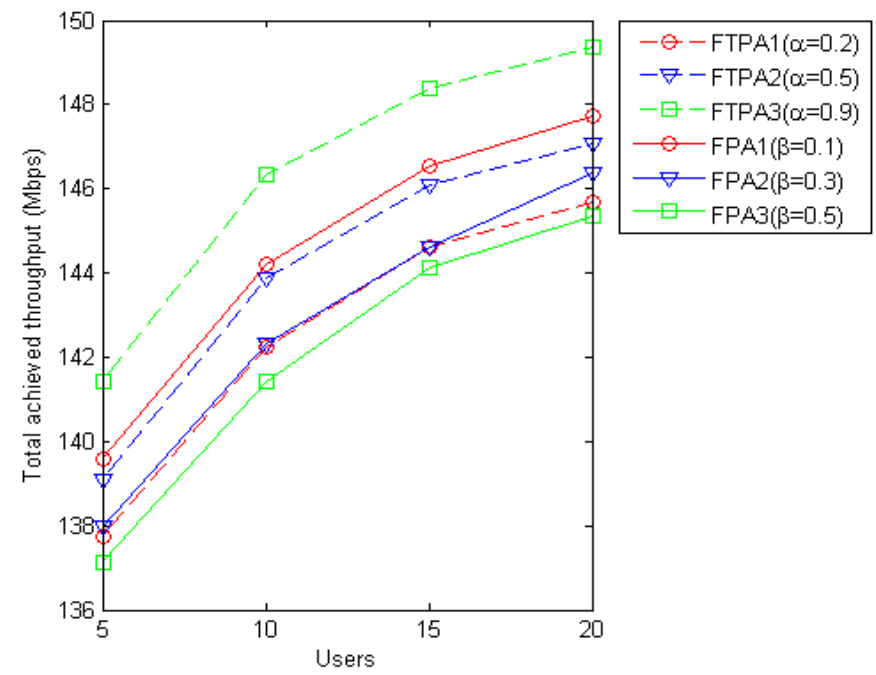

Fig. 5. PF-based NOMA's achieved cell throughput for all FPA and FTPA patterns.

At the first scheduling slot, i.e. $t=0$, none of the users has a historical rate. Consequently, and based on (6), the chosen user set is the set that has the highest feasible rate, i.e. with the highest $d$. At the second scheduling slot, i.e. $t=1$, user sets with lower $d$ are scheduled since they have lower historical rates than the user sets scheduled at $t=0$. The same goes on through the rest of the time slots, where user sets with low $d$ are chosen. The behavior of PF differs when FTPA is used since it does not have a unique sense of variation of the throughput: the throughput increases with the channel gain difference until reaching the critical point and then decreases with $d$, as seen in Fig. 4. At the first scheduling slot, the PF metric chooses the user sets with the highest feasible rates, which are situated in the region right before the critical point. From the second time slot, the choice is mostly limited to user sets with low $d$ or very high $d$, and the chosen set is the one with the highest feasible rate among possible candidate sets in these two regions. Given these points, the choice of user sets is expected to swing between the user groups with very low and very high values of $d$. We can conclude from this last analysis that the PF scheduler is probably not fully suited to NOMA schemes, since it doesn't allow users with significant channel gain difference to be paired together, while NOMA takes advantage of this difference to increase the achieved throughput for non-orthogonally scheduled users. Therefore, the use of the PF scheduler for NOMA should be questioned and different metrics for user assignment are still to be devised.

\section{CONCLUSION}

The performance of a NOMA system highly relies on the way users are paired together and on how the power is divided 
between users. In this work, we focused on these two steps by investigating the behavior of two types of intra-subband power allocation techniques, FPA and FTPA, and by evaluating the performance of the Proportional Fairness (PF) scheduler adopted in LTE. Results show that, first, the common belief that the increase in the channel gain difference is in favor of the throughput achieved by the NOMA scheme is not always verified: we showed it was confirmed for FPA but not for FTPA. Second, we observed that the PF scheduler most often multiplexes users with low channel gain difference, which limits the gains of NOMA. As a conclusion, in order to fully benefit from a given intra-subband technique, such as FPA, in a NOMA scheme, the user allocation technique has also to be reconsidered and jointly optimized with the intra-subband power allocation.

\section{APPENDIX}

This appendix describes the chain rule for computing the derivative of $R_{s, k_{2}}$ with respect to $d=h_{s, k_{1}}^{2}-h_{s, k_{2}}^{2}$, leading to (10).

After substitution by $P_{s, k_{1}}$ and $P_{s, k_{2}}, R_{s, k_{2}}$ can be rewritten as:

$$
R_{s, k_{2}}=B_{s} \log _{2}\left(1+\frac{P_{s} h_{s, k_{2}}^{2-2 \alpha}}{P_{s} h_{s, k_{1}}^{-2 \alpha} h_{s, k_{2}}^{2}+N_{s, k_{2}} h_{s, k_{1}}^{-2 \alpha}+N_{s, k_{2}} h_{s, k_{2}}^{-2 \alpha}}\right)
$$

where $N_{s, k_{1}}$ and $N_{s, k_{2}}$ are assumed to be equal.

Let $x=h_{s, k_{1}}$ and $y=h_{s, k_{2}}$ :

$$
\begin{gathered}
R_{s, k_{2}}=B_{s} \log _{2}\left(1+\frac{P_{s} y^{2-2 \alpha}}{P_{s} x^{-2 \alpha} y^{2}+N_{s, k_{2}} x^{-2 \alpha}+N_{s, k_{2}} y^{-2 \alpha}}\right) \\
\frac{d R_{s, k_{2}}}{d\left(x^{2}-y^{2}\right)}=\frac{d R_{s, k_{2}}}{d x} \times \frac{d x}{d\left(x^{2}-y^{2}\right)}+\frac{d R_{s, k_{2}}}{d y} \times \frac{d y}{d\left(x^{2}-y^{2}\right)} \\
=\frac{1}{2 x} \frac{d R_{s, k_{2}}}{d x}-\frac{1}{2 y} \frac{d R_{s, k_{2}}}{d y}
\end{gathered}
$$$$
\frac{d R_{s, k_{2}}}{d x}=\frac{u}{B_{s} \ln (2)\left(1+\frac{P_{s} y^{2-2 \alpha}}{P_{s} x^{-2 \alpha} y^{2}+N_{s, k_{2}} x^{-2 \alpha}+N_{s, k_{2}} y^{-2 \alpha}}\right)}
$$$$
u=\frac{2 \alpha P_{s}^{2} y^{4-2 \alpha} x^{-2 \alpha-1}+2 \alpha P_{s} N_{s, k_{2}} y^{2-2 \alpha} x^{-2 \alpha-1}}{\left(P_{s} x^{-2 \alpha} y^{2}+N_{s, k_{2}} x^{-2 \alpha}+N_{s, k_{2}} y^{-2 \alpha}\right)^{2}}
$$$$
\frac{d R_{s, k_{2}}}{d y}=\frac{v}{B_{s} \ln (2)\left(1+\frac{P_{s} y^{2-2 \alpha}}{P_{s} x^{-2 \alpha} y^{2}+N_{s, k_{2}} x^{-2 \alpha}+N_{s, k_{2}} y^{-2 \alpha}}\right)}
$$$$
v=\frac{(2-2 \alpha) P_{s} y^{1-2 \alpha}}{P_{s} x^{-2 \alpha} y^{2}+N_{s, k_{2}} y^{-2 \alpha}+N_{s, k_{2}} x^{-2 \alpha}}
$$$$
-\frac{P_{s} y^{2-2 \alpha}\left(2 y P_{s} x^{-2 \alpha}-2 \alpha N_{s, k_{2}} y^{-2 \alpha-1}\right)}{\left(P_{s} x^{-2 \alpha} y^{2}+N_{s, k_{2}} y^{-2 \alpha}+N_{s, k_{2}} x^{-2 \alpha}\right)^{2}}
$$

$$
\begin{aligned}
& \frac{d R_{s, k_{2}}}{d\left(x^{2}-y^{2}\right)} \\
& =\frac{\alpha P_{s}^{2} y^{4-2 \alpha} x^{-2 \alpha-2}+\alpha P_{s} N_{s, k_{2}} y^{2-2 \alpha} x^{-2 \alpha-2}+\alpha P_{s}^{2} y^{2-2 \alpha} x^{-2 \alpha}}{B_{s} \ln (2)\left(P_{s} x^{-2 \alpha} y^{2}+N_{s, k_{2}}\left(x^{-2 \alpha}+y^{-2 \alpha}\right)\right)\left(P_{s} x^{-2 \alpha} y^{2}+N_{s, k_{2}}\left(x^{-2 \alpha}+y^{-2 \alpha}\right)+P_{s} y^{2-2 \alpha}\right)} \\
& -\frac{P_{s} N_{s, k_{2}} y^{-4 \alpha}+(2-2 \alpha) P_{s} N_{s, k_{2}} y^{-2 \alpha} x^{-2 \alpha}}{B_{s} \ln (2)\left(P_{s} x^{-2 \alpha} y^{2}+N_{s, k_{2}}\left(x^{-2 \alpha}+y^{-2 \alpha}\right)\right)\left(P_{s} x^{-2 \alpha} y^{2}+N_{s, k_{2}}\left(x^{-2 \alpha}+y^{-2 \alpha}\right)+P_{s} y^{2-2 \alpha}\right)}
\end{aligned}
$$

To find the critical point of $R_{s, k_{2}}$, let $\frac{d R_{s, k_{2}}}{d\left(x^{2}-y^{2}\right)}=0$.

Hence, we obtain:

$\alpha P_{s}^{2} y^{4-2 \alpha} x^{-2 \alpha-2}+\alpha P_{s} N_{s, k_{2}} y^{2-2 \alpha} x^{-2 \alpha-2}+\alpha P_{s}^{2} y^{2-2 \alpha} x^{-2 \alpha}$

$-P_{s} N_{s, k_{2}} y^{-4 \alpha}-(2-2 \alpha) P_{s} N_{s, k_{2}} y^{-2 \alpha} x^{-2 \alpha}=0$

After simplifications and rearrangement:

$$
\alpha P_{s} y^{2}\left(\frac{y}{x}\right)^{2}+\alpha N_{s, k_{2}}\left(\frac{y}{x}\right)^{2}+\alpha P_{s} y^{2}+(2 \alpha-2) N_{s, k_{2}}=N_{s, k_{2}}\left(\frac{y}{x}\right)^{-2 \alpha}
$$

\section{REFERENCES}

[1] 5G-PPP Project on 5G Air Interface Below $6 \mathrm{GHz}$. Available on: http://fantastic5g.eu/wp-content/uploads/2015/09/EuCNCFANTASTIC-5G_final.pdf

[2] Y. Saito, Y. Kishiyama, A. Benjebbour, T. Nakamura, A. Li, and K. Higuchi. Non-Orthogonal Multiple Access (NOMA) for Future Radio Access. Vehicular Technology Conference (VTC Spring), pp.1-5, 2013.

[3] L. Dai, B. Wang, Y. Yuan, S. Han, C. I, and Z. Wang. Non-orthogonal multiple access for 5G: Solutions, Challenges, Opportunities, and Future Research Trends. IEEE Communications Magazine, vol. 53, no. 9, pp. 74-81, 2015.

[4] L.Anxin, L. Yang, C. Xiaohang, and J. Huiling. Non-orthogonal multiple access (NOMA) for future downlink radio access for $5 G$. China Communications, vol. 12, pp. 28-37, 2015.

[5] Z. Ding, M. Peng, and V. Poor. Cooperative Non-Orthogonal Multiple Access in $5 G$ Systems. IEEE Communications Letters, pp. 1462-1465, vol. 19 , no. 8, 2015.

[6] A. Benjebbour, A. Li, Y. Saito, Y. Kishiyama, A. Harada, and T. Nakamura. Concept and Practical Considerations of Non-orthogonal Multiple Access (NOMA) for Future Radio Access. Intelligent Signal Processing and Communications Systems (ISPACS), pp. 770-774, 2013.

[7] Z. Ding, P. Fan, and V. Poor. Impact of user pairing on $5 G$ nonorthogonal multiple access downlink transmissions. IEEE Transactions on Vehicular Technology, vol. PP, no. 99, pp. 1, 2015.

[8] Z. Ding, Z. Yang, P. Fan, and V. Poor. On the performance of nonorthogonal multiple access in $5 G$ systems with randomly deployed users. IEEE Signal Processing Letters, vol. 21, no. 12, pp. 1501-1505, 2014.

[9] S. Tomida and K. Higuchi. Non-orthogonal access with SIC in cellular downlink for user fairness enhancement. Intelligent Signal Processing and Communications Systems (ISPACS), pp. 1-6, 2011.

[10] Y. Saito, A. Benjebbour, Y. Kishiyama, and T. Nakamura. System-level Performance Evaluation of Downlink Non-orthogonal Multiple Access (NOMA). Personal Indoor and Mobile Radio Communications (PIMRC), pp. 611-615, 2013.

[11] M. Hojeij, J. Farah, C. Abdel Nour, and C. Douillard. New Optimal and Suboptimal Resource Allocation Techniques for Downlink Nonorthogonal Multiple Access. Wireless Personal Communications, vol. 82, no. 2, pp. 1-31, 2015.

[12] M. Kountouris and D. Gesbert. Memory-based Opportunistic Multi-user Beamforming Proceedings. International Symposium on Information Theory (ISIT), pp. 1426-1430, 2005.

[13] M. Kountouris and D. Gesbert. Memory-based Opportunistic Multi-user Beamforming. International Symposium on Information Theory (ISIT), pp. 1426-1430, 2005.

[14] Y. Bouguen, É. Hardouin, and F. Wolff, 2012. LTE et les réseaux $4 G$, Eyrolles. 